\title{
Pratiques
}

Linguistique, littérature, didactique

$169-170 \mid 2016$

Enseignement/apprentissage de la langue, des textes et des discours. 40\&nbspans de Pratiques

\section{Enseignement du lexique à l'école primaire et modélisations linguistiques : exemples d'activités portant sur des verbes de déplacement strict}

Vocabulary Teaching in Primary School and Linguistic Modelling: Examples of

Activities Based on Verbs of Strict Motion

Michel Aurnague et Claudine Garcia-Debanc

\section{(2) OpenEdition}

Journals

Édition électronique

URL : http://journals.openedition.org/pratiques/2990

DOI : $10.4000 /$ pratiques. 2990

ISSN : 2425-2042

Éditeur

Centre de recherche sur les médiations (CREM)

Référence électronique

Michel Aurnague et Claudine Garcia-Debanc, «Enseignement du lexique à l'école primaire et modélisations linguistiques : exemples d'activités portant sur des verbes de déplacement strict », Pratiques [En ligne], 169-170 | 2016, mis en ligne le 30 juin 2016, consulté le 10 décembre 2020. URL http://journals.openedition.org/pratiques/2990 ; DOI : https://doi.org/10.4000/pratiques.2990

Ce document a été généré automatiquement le 10 décembre 2020.

(c) Tous droits réservés 


\section{Enseignement du lexique à l'école primaire et modélisations linguistiques : exemples d'activités portant sur des verbes de déplacement strict}

Vocabulary Teaching in Primary School and Linguistic Modelling: Examples of Activities Based on Verbs of Strict Motion

Michel Aurnague et Claudine Garcia-Debanc

1 Dans son article de synthèse intitulé «La didactique du français et l'enseignement du vocabulaire dans vingt ans de revues de didactique du français langue première », É. Nonnon (2012) souligne combien «la question du vocabulaire et de son enseignement occupe, dans les travaux en didactique du français langue première, une place paradoxale », " en ce qu'elle est à la fois marginale [...] et affirmée d'une grande importance, tant par les enseignants que par les injonctions institutionnelles [...], les formateurs et les chercheurs " (Nonnon, 2012, p. 33). La mise en place des activités dont une partie des résultats est présentée dans cette contribution s'inscrit dans cette situation difficile.

\section{Contexte de l'étude : le lexique, mal aimé en didactique du français langue première ${ }^{1}$}

Depuis le numéro 43 de Pratiques, intitulé « Le sens des mots » (Masseron, 1984) et malgré la fécondité des évolutions théoriques dans les 30 dernières années (Grossmann, 2011), peu nombreuses ont été les recherches portant sur l'enseignement du lexique. Si les propositions didactiques concernant la phraséologie et les collocations se sont beaucoup développées en didactique de la L2 (Garcia-Debanc, Masseron \& Ronveaux, 2013), on 
n'observe pas la même dynamique en didactique du français langue première. Comment évaluer le vocabulaire d'un élève? Par quels dispositifs prendre en compte l'hétérogénéité entre les compétences lexicales des élèves? Quelle place respective réserver à l'enseignement occasionnel du lexique et à des séances d'étude de la langue ? Quel temps relatif consacrer à la morphologie, l'étymologie, l'orthographe, l'étude des relations sémantiques, au lexique-grammaire? Comment choisir le lexique à travailler à chacun des niveaux scolaires? Quelles activités proposer pour permettre l'acquisition et la mémorisation d'un lexique nouveau (Van der Linden, 2006) ? Telles sont quelques-unes des questions qui se posent à l'enseignant, au formateur et au chercheur en didactique.

En écho aux travaux psycholinguistiques mettant en évidence la souplesse et la plasticité des réseaux sémantiques dans le lexique mental (Van der Linden, 2006), nous avons choisi de travailler avec des élèves d'école primaire un réseau sémantique, celui des verbes de déplacement « autonome ». Les verbes de déplacement nous semblent en effet un contenu pertinent à travailler à l'école primaire, dans la mesure où les élèves sont fréquemment amenés à formuler des changements de localisation non seulement lorsqu'ils exposent à l'oral ou rédigent des récits mais aussi parfois en cours de sciences, lorsqu'ils ont à décrire les modes de déplacement d'animaux (Laparra, 2005). Notre étude se situe donc dans la perspective d'une articulation dynamique entre étude de la langue et production écrite : elle vise à constituer des aides lexicales (Garcia-Debanc, 2013) permettant aux rédacteurs de mobiliser des ressources lexicales en situation de production d'écrit.

La présente contribution se propose plus particulièrement d'interroger les articulations entre modélisations linguistiques en sémantique et données recueillies dans des classes de cycle 3 d'école primaire française (élèves de 9-12 ans). Cette articulation peut être considérée de divers points de vue. Du point de vue des recherches en sémantique, on peut se demander si les réponses des élèves permettent de valider le modèle sémantique de description des verbes de déplacement ou de l'affiner. Du point de vue psycholinguistique, les données recueillies permettent d'isoler les traces des connaissances et des intuitions d'enfants de 9 à 12 ans. Enfin, du point de vue des recherches en didactique du français langue première, le projet est d'évaluer les effets d'activités de classification de verbes de déplacement sur la diversité et la pertinence des emplois de ces verbes dans des récits, ce qui pose ainsi la question des effets des séances d'étude de la langue sur les compétences lexicales en production écrite. Toutefois, ce point ne sera pas abordé dans l'espace accordé à cette contribution (Garcia-Debanc \& Aurnague, 2015).

5 Les données analysées ici ont été collectées dans le cadre d'une recherche collaborative associant des enseignants d'école primaire et des formateurs, le projet «Didactique de la description du déplacement» (Didacdép) mené dans six classes de cycle 3, sous la coordination de notre collègue Michel Gangneux.

6 Nous présenterons successivement le protocole de recueil des données (section 2), la modélisation sémantique des verbes déplacement (section 3), l'analyse des productions orales et écrites des élèves, qui constituent les résultats (section 4), avant de proposer bilan et perspectives (section 5). 


\section{Protocole de recueil de données}

7 L'ingénierie didactique développée pour l'enseignement du lexique doit, selon nous, être guidée par un certain nombre de principes que nous avons déjà eu l'occasion de présenter en détail (Garcia-Debanc \& Chourau, 2010). Nous nous contenterons ici d'en rappeler quelques-uns. Il est, tout d'abord, nécessaire d'isoler, parmi les activités proposées aux enfants, des moments identifiés d'étude de la langue pendant lesquels s'articulent travail $d u$ lexique en langue et étude du lexique en discours, avec l'appui, notamment, des œuvres de littérature de jeunesse. Plus généralement, nous reprenons à notre compte les propositions d'A. Lieury (1997) visant à favoriser une approche « multimodale » des mots, qui combine production, réception, oral et écrit. L'appropriation de nouveaux items lexicaux par les élèves sera, en effet, d'autant plus stable que ceux-ci auront été appréhendés selon les dimensions complémentaires que déterminent ces modalités. Il y a, ensuite, un grand intérêt à aborder l'étude de nouveaux mots en s'appuyant sur le fonctionnement de lexèmes déjà connus ainsi qu'en mettant conjointement en lumière les propriétés sémantiques des items et leur comportement syntaxique. Du point de vue plus spécifique du sens, l'organisation des mots en grands domaines notionnels (espace, temps, sentiments...) permet l'exploration de sous-ensembles cohérents d'unités lexicales, à l'intérieur desquels pourront être mis en lumière les grands types de relations qui structurent le lexique - parmi lesquels la synonymie, l'antonymie, l'hypéronymie ou l'hyponymie (Cruse, 1986) - aussi bien que des relations plus rarement mentionnées telle la «troponymie» (relation entre verbes introduite par Miller \& Fellbaum, 1992) ${ }^{2}$. Enfin, on veillera à évaluer les connaissances des élèves avant et après la séquence d'enseignement.

8 Un protocole en six tâches, inspiré des principes précédents a été mis au point et proposé aux enseignants ayant pris part au projet Didacdép. Ce protocole comprenait :

- a) la rédaction individuelle de phrases intégrant des verbes de déplacement;

- b) la rédaction individuelle d'un texte narratif à partir du dessin d'animation Micro Loup de R. McGuire et $\mathrm{G}$. Solotareff ;

- c) la classification sémantique - avec explicitation des critères de regroupement - d'un ensemble de verbes de «changement de relation et d'emplacement " (liste A, section 3), activité qui débute par une classification individuelle, suivie d'une classification par groupes présentée sur affiches (après discussion et explicitation des critères) puis d'une mise en commun au sein de la classe ${ }^{3}$;

- d) la classification sémantique d'un ensemble de verbes de "changement d'emplacement " (liste B, section 3), selon la même procédure ;

- e) la réalisation d'activités ritualisées et, tout particulièrement, d'une tâche onomasiologique visant à produire ou éliciter des verbes sur la base d'un contenu sémantique donné (rituel « Léon »);

- f) la rédaction individuelle d'un texte narratif à partir d'un extrait du dessin d'animation Micro Loup, sans avoir sous les yeux le texte rédigé en tâche b.

Les résultats discutés plus loin sont nécessairement restreints et se focalisent, pour l'essentiel, sur la rédaction individuelle de phrases (tâche a ci-dessus) ainsi que sur la classification de verbes de déplacement strict («changements de relation et d'emplacement ») de la liste A (tâche c). Les productions ont été recueillies dans trois classes de Toulouse et sa banlieue dont les élèves provenaient de milieux sociaux 
hétérogènes: classe de Sophie (CE2A, banlieue toulousaine), classe de Chantal (CE2B, Toulouse) et classe de Lucile (CM1-CM2, banlieue toulousaine). Ajoutons que, tout en préservant la cohérence globale de la trame détaillée plus haut, une certaine liberté a été laissée aux enseignants au moment d'implémenter le protocole d'étude des verbes de déplacement.

\section{Les verbes de déplacement : modélisation linguistique}

Comme il a déjà été évoqué, les activités proposées aux élèves dans le cadre du projet Didacdép sont basées sur une modélisation sémantique fine des items linguistiques mobilisés et, tout particulièrement, des verbes de déplacement. Les prédicats spatiaux dynamiques auxquels nous nous intéressons sont des verbes intransitifs - transitifs « indirects » inclus - décrivant le déplacement " autonome » d'une entité mobile (seuls quelques verbes transitifs directs apparaissent dans les activités : atteindre, quitter) : aller à , arriver, déraper, descendre, entrer, foncer, se hisser, partir, ramper, se rendre, sortir... Ils se distinguent à la fois syntaxiquement et sémantiquement des prédicats spatiaux dynamiques (généralement transitifs "directs») dénotant un déplacement dont le caractère "causé » ou induit est rendu explicite par l'identification (via le sujet syntaxique) de l'agent à l'origine du procès : amener, apporter, conduire, emporter, trainer, transporter...

11 Depuis au moins deux décennies, les travaux syntacticosémantiques sur l'expression du déplacement dans la langue sont parcourus par une opposition récurrente entre les verbes de déplacement autonome véhiculant la manière de se mouvoir et ceux qui indiquent un déplacement au sens strict (ex: Jackendoff, 1990 ; Levin, 1993; Levin \& Rappaport, 1992) : foncer, grimper, marcher, zigzaguer versus arriver, partir, sortir, se rendre. Le recours à cette distinction trouve sans doute son origine dans le contraste fondateur établi par L. Talmy (1985) entre l'expression de la manière du déplacement et celle du trajet ou «path». De nombreuses analyses linguistiques ont tenté de saisir l'opposition entre manière et déplacement strict via l'aspect interne ou mode d'action des verbes (ex : Dini \& Di Tomaso, 1999) : alors que la première notion donnerait naissance à des procès atéliques - pouvant se prolonger indéfiniment puisque non pourvus de borne ou transition intrinsèque : foncer, grimper, marcher, zigzaguer -, la seconde se matérialiserait dans des prédicats transitionnels qui mettent, par conséquent, en jeu la télicité - ex: arriver, partir, sortir, se rendre. Le traitement aspectuel de la distinction mentionnée cidessus n'est pas sans poser problème (Aurnague, 2012) et nous avons, pour notre part, préféré en rendre compte en recourant à des concepts spatiotemporels qui seront, dans un second temps, mis en relation avec le comportement des prédicats en termes de mode d'action.

12 Pour autant, la mise au jour de concepts spatiotemporels appropriés n'est pas une tâche aisée, comme le montrent les problèmes que soulève une caractérisation des verbes de déplacement strict en termes de changement de lieu (Laur, 1991; Randall, 2010) ou de déplacement dirigé inhérent (Levin, 1993). Alors que la première notion catégorise comme des «lieux » des entités qui n'en sont pas (et tend à réduire la sémantique des relations spatiales à une relation géométrique d'inclusion dans une région), la seconde recourt à un critère qui n'est non seulement pas exclusif des prédicats de déplacement 
strict, mais semble vérifié par peu d'entre eux (pour plus détails, voir Aurnague, 2008, 2011). Une définition spatiotemporelle des verbes et procès de déplacement est cependant possible. Elle doit, selon nous, faire appel aux deux notions distinctes que sont le changement d'emplacement et le changement de relation locative élémentaire. Une entité-cible ou entité localisée participe à un changement d'emplacement (également dénommé «translocation" dans d'autres travaux) si sa position dans le cadre de référence terrestre varie au cours du procès considéré: contrairement à s'assoir qui n'implique pas de déplacement dans le cadre de référence englobant, marcher est bien porteur de cet état de fait. La notion de changement de relation locative élémentaire due à J. P. Boons (1987) suppose, quant à elle, que la relation entre une entité-cible et l'entitésite par rapport à laquelle s'opère la localisation soit modifiée durant le procès: les énoncés Max a marché dans la prairie et Max est entré dans la prairie ont ceci de différent que seul le second met en jeu un changement de relation locative (être dans) par rapport au site désigné par le groupe nominal la prairie (voir, plus loin, la schématisation du verbe entrer sur la base de la relation être dans).

Les deux concepts ainsi mis en évidence dessinent une combinatoire complexe à partir de laquelle quatre catégories de verbes et procès peuvent être définies (voir tableau 1). Les changements d'emplacement sans changement de relation, évoqués plus haut à propos de marcher, regroupent, en particulier, les verbes de déplacement généralement considérés comme relevant de la manière. À l'opposé, un changement de relation locative élémentaire peut intervenir sans que soit impliqué aucun changement d'emplacement, ce qui se produit, par exemple, pour les prédicats sous-tendus par la relation spatiale de support/contact (être sur; ex: se poser). Les verbes de déplacement strict qui sont au centre de notre modélisation linguistique combinent, comme on peut le constater, un changement de relation et un changement d'emplacement (voir ci-dessous). Enfin, certains verbes et procès ne mobilisent aucune des deux notions utilisées : appartiennent (entre autres) à cette catégorie la plupart des prédicats de changement de posture (ex: s'accroupir, s'assoir, se recroqueviller) ainsi que les verbes décrivant la façon spécifique dont l'entité-cible « occupe » le site (ex : se blottir, se cacher, s'embourber, se ficher).

Tableau 1. Catégories de verbes et procès de mouvement/déplacement

\begin{tabular}{|l|l|l|}
\hline & Changement d'emplacement & Pas de changement d'emplacement \\
\hline $\begin{array}{l}\text { Pas de changement } \\
\text { de relation }\end{array}$ & $\begin{array}{l}\text { ex: avancer, foncer, grimper } \\
\text { marcher, nager, patrouiller } \\
\text { zigzaguer } \\
\text { (cf. liste B) }\end{array}$ & $\begin{array}{l}\text { ex : s'accroupir, s'asseoir, se recroqueviller } \\
\text {; se blottir, se cacher, s'embourber, se ficher }\end{array}$ \\
\hline $\begin{array}{l}\text { Changement de } \\
\text { relation }\end{array}$ & $\begin{array}{l}\text { ex: aller + Prép., arriver, partir, } \\
\text { sortir, se rendre } \\
\text { (cf. liste A) }\end{array}$ & $\begin{array}{l}\text { ex : se poser [toucher, frôler] ; s'immerger ; } \\
\text { sauter, bondir }\end{array}$ \\
\hline
\end{tabular}

Au-delà des catégories de verbes ou procès que les deux concepts retenus permettent de circonscrire, l'interaction entre changements de relation et changements d'emplacement doit être observée à l'intérieur même de la classe de prédicats qui associe ces concepts, à savoir les verbes de déplacement strict. Le sémantisme de ces verbes (ex : aller + Prép., 
arriver, partir, sortir, se rendre) fait apparaitre deux schémas spatiotemporels principaux (Aurnague, 2008, 2011). Le changement de relation et le changement d'emplacement peuvent, tout d'abord, coïncider temporellement, comme cela se produit pour entrer et sortir, dont la sémantique, basée sur la relation spatiale être dans (changement de relation), se double d'un changement d'emplacement concomitant. Dans d'autres cas, les deux changements sont temporellement disjoints, le changement de relation par rapport au site étant précédé d'un changement d'emplacement (ex: aller à, se rendre). Une "polarité " peut être associée à chaque verbe de déplacement strict, en fonction de la structure du changement de relation sous-jacent. Elle est initiale lorsque la relation est assertée puis niée (l'information « positive » est première : $r \cdots \triangleright \neg$; ex. : sortir, être-dans

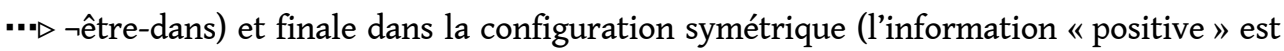
seconde : $\neg \mathrm{r} \cdots \triangleright r$; ex. : entrer, $\neg$ être-dans $\cdots \triangleright$ être-dans). On parle, de la même façon, de polarité médiane - assertion d'une relation précédée et suivie de sa négation : $\neg \mathrm{r} \cdots \triangleright \mathrm{r} \cdots$ $\triangleright \neg r$ - mais celle-ci concerne un très petit nombre de verbes et locutions verbales $d u$ français (ex. : couper par, passer par) et n'intervient pas dans les activités proposées aux élèves ${ }^{4}$.

La modélisation linguistique dont nous venons d'esquisser les contours a permis, on le voit, de dégager un nombre important d'éléments de sens intervenant dans l'élaboration des procès de déplacement : cible (entité localisée), site (entité localisatrice), changement d'emplacement, changement de relation locative élémentaire, polarité, vitesse (cf. note 4), opposition à une force (note 4), direction (note 4), entrainement par une force (note 4)... L'un des objectifs théoriques des activités imaginées dans le cadre du projet Didacdép était précisément de tester le bienfondé de ces notions et leur utilisation/ maniement par les élèves. Sur un plan psycholinguistique, nous faisons l'hypothèse que la mise en place de tâches de natures diverses (rédaction de textes narratifs, production de phrases, classification/tâche sémasiologique, rituel/tâche onomasiologique) devrait s'accompagner d'accès contrastés aux notions sémantiques que véhiculent les verbes et éclairer ainsi l'étude des processus cognitifs associés. Deux listes ont été soumises aux enfants pour les activités de classification, l'une constituée de verbes de changement de relation et d'emplacement (déplacement strict; listeA) et l'autre contenant uniquement des verbes de changement d'emplacement (liste B):

- aller (à), s'en aller, aboutir, s'échapper, accéder, s'enfuir, arriver, entrer, partir, parvenir, pénétrer, se rendre, sortir, venir, atteindre, fuir, quitter (liste A) ;

- avancer, couler, courir, dégringoler, déraper, descendre, foncer, dévaler, glisser, grimper, ramper, se hisser, reculer, rouler, monter, se traîner, marcher, nager, patiner, skier (liste B).

Dans la suite de ce travail, nous nous concentrerons sur les activités de production de phrases et de classification de verbes incluses dans le protocole de recueil de données (cf. section 2) et, plus spécifiquement encore, sur celles qui mettent en jeu la liste A développée ci-dessus.

\section{Résultats}

17 Conformément à ce qui a été indiqué, les résultats synthétisés dans cette section portent sur deux classes de CE2 (CE2A : banlieue toulousaine, CE2B : Toulouse) et une classe de CM1-CM2 (banlieue toulousaine). Après avoir examiné la production de phrases par les élèves (section 4.1), nous nous pencherons sur les classifications obtenues pour les verbes de déplacement strict (section 4.2). 


\subsection{Production de phrases}

CM2. Le nombre de verbes de déplacement autonome (différents) utilisés par élève a été plus élevé en CE2A qu'en CM1-CM2 mais ce décalage est directement imputable au contenu de la consigne qui proposait, dans le premier cas, mais non dans le second, la rédaction de plusieurs phrases (contenant, le cas échéant, plusieurs verbes) ${ }^{5}$. Pour ce qui est de la nature des verbes élicités, deux différences principales apparaissent entre les classes. On note, tout d'abord, que les prédicats de déplacement au sens strict ne représentent que 29,4\% des occurrences de verbes produites en CE2A (arriver (1), partir (1), entrer/rentrer (2), passer + Prép. (2), aller + Prép. (4), se rapprocher (5), soit 15 occurrences sur un total de 51) alors qu'on dénombre 66,7 \% de productions de ce type en CM1-CM2 ( s'enfuir (1), plonger (1), rentrer (1), retourner (1), venir (1), s'échapper (3), partir (4), aller + Prép. (14), soit 26 occurrences sur 39$)^{6}{ }^{6}$. En d'autres termes, les CM1-CM2 illustrent massivement la catégorie sémantique du déplacement au moyen de déplacements stricts (plutôt qu'en recourant aux changements d'emplacement et à la manière comme le font les CE2A) et accordent, en outre, une importance spécifique au verbe aller (+ Prép.; ex : aller à) identifié par Å. Viberg (2002) comme étant un « verbe basique » pour l'expression de l'espace dynamique. L'autre différence observée a trait à la production, par les CE2A, de plusieurs constructions associant le verbe support faire à un nom d'activité ou d'instrument (ex: faire de la luge/de l'équitation/des pas chassés/du sport/du vélo), ces occurrences étant, semble-t-il, le fait d'élèves confrontés à des difficultés plus générales.

La production « dirigée » diffère de la production libre par le recours imposé à des items lexicaux prédéfinis (liste A). Cette contrainte supplémentaire n'est pas neutre et conduit parfois les élèves à élaborer des phrases ne relevant pas véritablement du déplacement autonome, soit qu'ils n'aient pas (encore) totalement saisi cette notion sémantique, soit qu'ils peinent à correctement distinguer les divers usages des items verbaux listés ${ }^{7}$. Sans exclure l'étude ultérieure et/ou indépendante d'autres emplois des verbes (emplois «métaphoriques » notamment), c'est pourtant bien au déplacement « concret » d'entités matérielles que les élèves ont été majoritairement confrontés depuis le début du protocole. Quatre types de productions qui n'étaient pas attendues ont pu être recensés. Ainsi, alors que certains verbes endossent une fonction d'auxiliaire aspectuel (ex : venir indiquant le passé proche ; voir (1) plus bas), d'autres prennent une valeur quasi modale ( parvenir $=$ réussir à) ou apparaissent dans des contextes n'impliquant pas le déplacement spatial concret d'une entité-cible autonome (aboutir, atteindre: 2). Dans d'autres situations, c'est la polysémie du verbe qui fait problème (se rendre: 3 ) ou son insertion dans des constructions - spatiales ou non - autres que celles visées (rendre, fuir : 4). 
Comme on peut le constater dans les exemples ci-dessous, ces productions « décalées » se retrouvent aussi bien en CE2A qu'en CM1-CM2 et ne sont donc pas l'apanage d'une classe ou d'un niveau particulier8.

(1) Nous venons de sortir de la maison (CE2A); Il vient de te voir (CM1-CM2)9

(2) Je parviens à attraper ce livre (CE2A); Elle parvient à marcher (CM1-CM2); L'enquête

aboutit au coupable (CE2A); Il a atteint son objectif (CM1-CM2)

(3) Les voleurs se rendre à la police (CE2A); Il s'est rendu quand la police l'a coincé (CM1-

$\mathrm{CM} 2)$

(4) Je te rends ta voiture (CE2A); Le robinet fuit (CE2A)

Un autre phénomène remarquable émerge de l'activité dirigée de production de phrases mais il concerne, cette fois, des énoncés pour la plupart conformes à la consigne donnée, en ce sens qu'ils réfèrent bien à des situations de déplacement autonome (déplacement « fictif » inclus; voir Talmy, 2000). Ce qui fait la spécificité des productions mises au jour est donc ailleurs : des verbes dont le modèle théorique prédit la proximité sémantique aboutir, accéder, parvenir - (voir plus loin), donnent lieu au(x) même(s) type(s) de constructions syntaxiques "erronées » (5-7). Les écarts (syntaxiques) relevés tiennent, par exemple, à la nature de l'auxiliaire utilisé (ex: Le chemin est abouti ; J'ai parvenu chez mes amis) et/ou à l'insertion inappropriée de pronoms personnels ou réfléchis (ex: Le chemin m'a abouti à la maison; Le chemin s'aboutit (à l'école); Nous nous sommes accédés à la ferme). Mais c'est sans doute le recours à des constructions transitives (directes) qui frappe le plus lorsque l'on examine les données relatives à ces trois verbes: Nous avons abouti la route; J'accède la maison; Je parviens la tour Eiffel (cf. (5-7)) ${ }^{10}$. Avec arriver, les prédicats aboutir, accéder et parvenir forment la classe des changements de relation finaux avec déplacement antérieur présupposé (le verbe transitif atteindre appartient au même groupe ; Aurnague, 2008, 2011). Tout en présentant un même schéma spatiotemporel, ces verbes diffèrent par un certain nombre de traits susceptibles d'être incorporés à leur sémantisme (Aurnague, 2015) : guidage, obstacle/difficulté " externe", intentionnalité versus non-intentionnalité ${ }^{11}$. La présence de ces éléments de sens additionnels et les conséquences qui s'ensuivent en termes d'" affectation » de l'entité-site (localisatrice) et/ ou de la cible (localisée) impliquées dans le procès confèrent à ce dernier un haut degré de "transitivité sémantique » (Hooper \& Thompson, 1980 ; Sarda, 1999) ; ce qui explique, entre autres, l'apparition d'emplois transitifs (directs) dans les données recueillies ${ }^{12}$. Les écarts constatés sont donc prévisibles du point de vue du modèle linguistique alors qu'ils le seraient moins si l'on se limitait à des données de surface telles la fréquence des items lexicaux concernés dans les manuels scolaires ${ }^{13}$.

(5) Le chemin est abouti (CE2A); Le chemin m'a abouti à la maison (CE2A); Le chemin s'aboutit à l'école (CE2A); La rue s'aboutit (CM1-CM2) ; La rue s'aboutit à la mer (CM1$\mathrm{CM} 2)$; La route aboutit la mer (CE2A); ...aboutit la maman (CE2A); Vous avez abouti la glace (CM1-CM2); Nous avons abouti la route (CM1-CM2)

(6) Nous nous sommes accédés à la ferme (CM1-CM2); J'accède la porte du magasin (CE2A) ; J'accède la maison (CE2A); Elle a accédé la porte (CM1-CM2)

(7) J'ai parvenu chez mes amis (CM1-CM2); On a parvenu à la forêt (CM1-CM2); Je parviens la maison (CE2A); Je parviens la tour Eiffel (CE2A); Je parviens enfin mon stylo (CE2A)

Les emplois non attendus des verbes de la liste A et les principaux écarts syntaxiques étant recensés, il est possible de mieux délimiter les productions "appropriées" recueillies dans chaque classe. Quel que soit le niveau (CE2A ou CM1-CM2), il apparait qu'une majorité d'élèves utilisent « correctement » entre 11 et 15 des 17 verbes proposés, c'est-à-dire au moins deux tiers des items (voir Tableau 2). Une observation plus fine 
révèle cependant des différences entre les deux classes. Ainsi, alors qu'en CE2A, seulement 5 des 17 verbes donnent lieu à plus de $80 \%$ de productions appropriées par (plus de) $80 \%$ d'élèves - aller à, arriver, s'échapper, partir, pénétrer -, le nombre de lexèmes remplissant la même contrainte se porte à 12 (sur 17) en CM1-CM2 - s'en aller, s'enfuir, entrer, fuir, quitter, sortir et venir s'ajoutent aux verbes cités ci-dessus ${ }^{14}$. Que cela soit dû à une meilleure appréhension du concept de déplacement autonome ou à une connaissance accrue du fonctionnement des verbes, on constate donc une plus grande maitrise de la production de phrases dirigée chez les élèves les plus âgés, à savoir ceux de CM1-CM2.

Tableau 2. Répartition des élèves par fourchettes d'emplois « appropriés »

\begin{tabular}{|l|l|l|l|l|}
\hline Nombre d'emplois « appropriés » & $0-5$ & $6-10$ & $11-15$ & $16-17$ \\
\hline Nombre d'élèves CE2A & 2 & 3 & 12 & 4 \\
\hline Nombre d'élèves CM1-CM2 & 1 & 4 & 14 & 3 \\
\hline
\end{tabular}

\subsection{Classification des verbes de déplacement strict} plusieurs étapes successives, depuis le travail individuel jusqu'à la mise en commun en passant par des échanges au sein de groupes d'élèves. Nous examinons, dans la suite, les classifications individuelles recueillies en CE2A puis les comparons à celles dégagées par les divers groupes du CE2B.

21 élèves du CE2A ont pris part à l'activité de classification individuelle (consigne: «Propose un classement des verbes suivants...»), 2 écrits sur 23 ne portant la trace d'aucun résultat. Parmi les 19 classifications élaborées :

- 12 s'appuient sur l'opposition entre verbes de polarité initiale et finale (agrémentée de raffinements éventuels ; voir plus loin) ;

- 4 utilisent ce même contraste mais comportent, en outre, plusieurs classes de verbes « incohérentes » ou « résiduelles »;

- enfin, 3 classifications sont entièrement incohérentes ou mobilisent des critères qui ne nous ont pas paru interprétables.

Trois types principaux d'entêtes ou labels ont été imaginés par les élèves pour mettre en regard les verbes de polarité initiale versus finale :

- partir vs venir : quand tu pars vs quand tu viens, je pars vs venir, partir vs venir ;

- partir/s'en aller vs aller à : partir de quelque part vs aller quelque part, je m'en vais de quelque part vs je vais quelque part ;

- sortir vs entrer.

L'examen du nombre de verbes classés par élève (tableau 3) fait apparaitre une répartition à peu près égale entre les élèves qui ont traité 10 items lexicaux au plus sur 17 (2 à $10: 9)$ et ceux qui ont franchi ce seuil (11 à $17: 10)$. D'autre part, il est instructif de croiser ces données avec celles relatives à la nature des productions : on remarque, ce faisant, que 5 des 9 classifications de 2 à 10 verbes sont incohérentes ou partiellement incohérentes $(55,55 \%)$ alors que seules 2 des 10 classifications impliquant 11 à 17 verbes 
présentent cette même particularité ( $20 \%)$. Il en résulte que les élèves qui traitent le plus de verbes sont aussi les plus aptes à dégager des classifications cohérentes.

Tableau 3. Répartition des élèves de CE2A en fonction du nombre verbes classés

\begin{tabular}{|l|l|l|l|l|}
\hline Nombre de verbes classés & $2-5$ & $6-10$ & $11-15$ & $16-17$ \\
\hline Nombre d'élèves & 4 & 5 & 6 & 4 \\
\hline
\end{tabular}

Comme les classifications individuelles de la classe de CE2A, celles réalisées en groupes par les élèves du CE2B (7 groupes) se fondent massivement sur l'opposition entre verbes initiaux et finaux. Les entêtes ou labels ayant été choisis par les élèves sont les suivants :

- partir vs aller vers : partir de quelque part vs aller vers quelque part

- $\rightarrow$ vs $\rightarrow$ (représentation graphique)

- s'échapper vs \{ aller (à) [aller à, se rendre, venir] + être [aboutir, accéder, arriver, atteindre, parvenir (, entrer)]\}.

Hormis une production incohérente (formée de 6 sous-ensembles), les propositions des autres groupes d'élèves s'appuient sur 2 catégories de verbes - contraste initial versus final - ou, plus souvent encore, sur 3 catégories (voir le troisième entête/label ci-dessus). Cette dernière configuration mérite une attention particulière car, si elle illustre la possibilité (pointée plus haut) de raffiner l'opposition initial versus final, elle met aussi en lumière la finesse des intuitions sémantiques auxquelles ont accès les enfants. Comme on peut le constater, ces intuitions les conduisent à dissocier, au sein de la catégorie des verbes ou procès finaux, ceux qui, du point de vue de l'aspect interne ou mode d'action, présentent les caractéristiques d'un « accomplissement » (aller à, se rendre, venir...) et ceux qui se comportent davantage comme des "achèvements" (aboutir, accéder, arriver, atteindre, parvenir, entrer...).

\section{Bilan et perspectives}

Les résultats présentés dans cette contribution doivent être associés à l'analyse des données issues du traitement de la liste B (verbes de changement d'emplacement, section 3) par les élèves de cycle 3, aussi bien dans leur versant classificatoire/ sémasiologique que dans leur versant onomasiologique ritualisé (Aurnague \& GarciaDebanc, 2016). Cet ensemble d'observations tend à démontrer la capacité des élèves à manipuler et maitriser des propriétés de sens qui apparaissent, au premier abord, relativement abstraites et d'une certaine complexité, telles la polarité, largement mobilisée pour la classification des verbes de la liste A (section 4.2). Les données recueillies confortent, plus généralement, les bases du modèle utilisé pour concevoir les activités d'enseignement et illustrent les bénéfices que peut retirer l'ingénierie didactique de travaux préalables de linguistique théorique. Elles montrent également l'importance du corpus soumis à l'observation des élèves pour leur donner l'occasion d'expliciter leurs intuitions sémantiques. En effet, dans un travail antérieur conduit avec des élèves des mêmes niveaux scolaires utilisant un corpus moins ciblé, le critère de polarité apparaissait de façon moins saillante (Garcia-Debanc et al., 2009). 

élèves en relation avec la liste $\mathrm{A}$ de verbes de changement de relation et d'emplacement concerne les liens qui s'établissent entre certaines propriétés de sens des prédicats verbaux et les constructions syntaxiques dans lesquelles ceux-ci peuvent entrer. Ces liens sont parfois extrêmement profonds et leur prédiction par le modèle peut amener à comprendre, et peut-être même anticiper, des productions " déviantes » (relativement à la norme et à ce qui est réellement « réalisé » dans la langue) qui seraient sinon traitées au même titre que d'autres "erreurs"; on a vu ainsi, à la section 4.1, les emplois transitifs de certains verbes indiquant des changements de relation finaux: aboutir, accéder, parvenir.

L'analyse complète du corpus Didacdép suppose, bien sûr, qu'une attention spécifique soit portée aux tâches narratives intégrées au début et à la fin du protocole (voir étapes $b$ et $f$ à la section 2). Ce n'est qu'alors que pourront être appréhendés les possibles effets des activités - de classification notamment - sur l'emploi de verbes en production écrite (Garcia-Debanc \& Aurnague, 2015).

Un examen approfondi de l'ingénierie didactique devra, à plus longue échéance, être également réalisé, afin de déterminer les écarts entre les activités initialement imaginées par les chercheurs et leur mise en œuvre par les enseignants et tenter de cerner, de la sorte, les différentes conceptions de l'enseignement du lexique que ces écarts révèlent.

\section{BIBLIOGRAPHIE}

AURNAGUE, M. (2008). «Qu'est-ce qu'un verbe de déplacement ? : critères spatiaux pour une classification des verbes de déplacement intransitifs du français ». In : Durand, J., Habert, B. \& Lacks, B. (éds), Actes du Congrès mondial de linguistique française, CMLF'08. Paris : ILF/EDP Sciences, p. 1905-1917 (cd-rom). En ligne : http://dx.doi.org/10.1051/cmlf08041.

- (2011). « How motion verbs are spatial: the spatial foundations of intransitive motion verbs in French ». Lingvisticae Investigationes 1, 34, p. 1-34.

- (2012). « De l'espace à l'aspect : les bases ontologiques des procès de déplacement ». Corela HS-12. En ligne : http://corela.revues.org/2846.

- (2015). « Motion verbs and spatial PPs in French: from spatio-temporal structure to asymmetry and goal bias ». Carnets de Grammaire 23, rapport CLLE-ERSS.

AURNAGUE, M. \& GARCIA-DEBANC, C. (2016). « Les verbes de déplacement comme contenu d'enseignement du lexique à l'école primaire : modélisation linguistique et analyse de productions d'élèves ». In : Neveu, F., Bergounioux, G., Côté, M. H., Fournier, J. M., Hriba, L. \& Prévost, S. (éds), Actes du $5^{e}$ Congrès mondial de linguistique française, CMLF 2016. Paris : ILF/EDP Sciences. En ligne : http://dx.doi.org/10.1051/shsconf/20162707001.

BOONS, J. P. (1987). « La notion sémantique de déplacement dans une classification syntaxique des verbes locatifs ». Langue française 76, p. 5-40.

CRUSE, D. A. (1986). Lexical semantics. Cambridge : Cambridge University Press. 
DINI, L. \& DI TOMASO, V. (1999). « Linking theory and lexical ambiguity: the case of Italian motion verbs ». In : Bunt, H. \& Muskens, R. (eds), Computing meaning, vol. 1. Dordrecht : Kluwer, p. 321-337.

GANGNEUX, M. \& GARCIA-DEBANC, C. (2013). Enseigner le lexique au cycle 3 : l'exemple des verbes de déplacement (2 DVD vidéo, 1 cédérom). Toulouse : IUFM Midi-Pyrénées.

GARCIA-DEBANC, C. (2013). «Les aides lexicales à la rédaction de textes : panorama historique et essai de typologie ». In : Garcia-Debanc, C., Masseron, C. \& Ronveaux, C. (éds), Enseigner le lexique. Namur : Presses universitaires de Namur, p. 273-300.

Garcia-Debanc, C. \& aurnague, M. (2015). « Effets d'un module d'enseignement des verbes de déplacement sur l'emploi de verbes spécifiques dans des productions écrites narratives à l'école primaire ». Présentation au 83 congrès de l'ACFAS, 23-25 mai, Université du Québec à Rimouski.

GARCiA-DEBANC, C. \& CHOURAU, A. (2010). « Enseigner les verbes de déplacement pour l'écriture de récits : de l'analyse de besoins à la mise en place d'activités en classe de CM2 ». Recherches 53, p. 139-158.

GARCiA-DEBANC, C., DUVignaU, K., DUtRAit, C. \& GANGNeuX, M. (2009). « Enseignement du lexique et production écrite. Un travail sur les verbes de déplacement à la fin de l'école primaire ». Pratiques 141-142, p. 208-232.

Garcia-Debanc, C., MASSERon, C. \& RonVeaux, C (éds) (2013). Enseigner le lexique. Namur : Presses universitaires de Namur.

Grossmann, F. (2011). « Didactique du lexique : état des lieux et nouvelles orientations ». Pratiques 149-150, p. 163-183.

HOOPER, P. J. \& THOMPSON, S. A. (1980). « Transitivity in grammar and discourse ». Language 56-2, p. 251-299.

JACKENDOFF, R. (1990). Semantic structures. Cambridge: MIT Press.

LAPARRA, M. (2005). «Capacités langagières en production non fictionnelle ». Pratiques 125-126, p. $139-156$.

LEVIN, B. (1993). English verb classes and alternations: a preliminary investigation. Chicago: The University of Chicago Press.

LEVIN, B. \& RAPPAPORT, M. (1992). « The lexical semantics of verbs of motion: the perspective from unaccusativity ». In: Roca, I. M. (ed.), The thematic structure: its role in grammar. Berlin : Foris Publications, p. 247-269.

LAUR, D. (1991). Sémantique du déplacement et de la localisation en français : une étude des verbes, des prépositions et de leurs relations dans la phrase simple. Thèse de doctorat, Université de Toulouse-Le Mirail.

LIEURY, A. (1997). Mémoire et réussite scolaire. Paris : Dunod.

MASSERON, C. (éd.) (1984). « Le sens des mots ». Pratiques 43.

Miller, G. \& fellbaum, C. (1992). « Semantic networks in English ». In : Levin, B. \& Pinker, S. (eds), Lexical \& conceptual semantics. Oxford: Blackwell, p. 197-229.

NONNON, E. (2012). « La didactique du français et l'enseignement du vocabulaire dans vingt ans de revues de didactique du français langue première ». Repères 46, p. 33-72. 
ORTÉGA, E \& LÉTÉ, B. (2010). eManulex: electronic version of Manulex and Manulex-Infra databases. En ligne : http://www.manulex.org.

RANDALL, J. H. (2010). Linking: the geometry of argument structure. Dordrecht: Springer.

SARDA, L. 1999. Contribution à l'étude de la sémantique de l'espace et du temps : analyse des verbes de déplacement transitifs directs du français. Thèse de doctorat, Université Toulouse 2-Le Mirail.

TALMY, L. (1985). « Lexicalization patterns: semantic structure in lexical forms ». In: Shopen, T. (ed.), Language typology and syntactic description, vol. 3, Grammatical categories and the lexicon. Cambridge: Cambridge University Press, p. 57-143.

TALMY, L. (2000). Toward a cognitive semantics, vol. 1 \& 2. Cambridge: MIT Press.

TLF (1971-1994). Trésor de la langue française, version informatisée (TLFi, 2004). Nancy : Atilf/CNRS. En ligne : http://atilf.atilf.fr/tlfi.htm.

VANDELOISE, C. (2001). Aristote et le lexique de l'espace: rencontres entre la physique grecque et la linguistique cognitive. Stanford : CSLI.

VAN DER LINDEN, E. (2006). « Lexique mental et apprentissage des mots ». Revue française de linguistique appliquée 1, vol. XI, p. 33-44.

VIBERG, Å. (2002). « Basic verbs in second language acquisition ». Revue française de linguistique appliquée VII, 2, p. 51-69.

\section{NOTES}

1. Nous remercions les collègues de l'Espé de l'académie de Toulouse et les professeurs des écoles ayant pris part à la conception des activités évoquées dans cette étude et à leur mise en œuvre dans des classes. Ce travail a été réalisé dans le cadre d'une recherche collaborative Didacdép soutenue par l'Institut universitaire de formation des maitres (IUFM) Midi-Pyrénées. Notre reconnaissance va plus spécialement à Michel Gangneux, enseignant formateur à l'ESPE, qui a coordonné la captation vidéo de diverses séances dans les classes et l'édition du double dvd Enseigner le lexique au cycle 3 : l'exemple des verbes de déplacement qui en émane (Gangneux \& GarciaDebanc, 2013). Nous remercions également les deux relecteurs anonymes ayant évalué une version précédente de ce texte pour leurs remarques précises et constructives.

2. La relation de troponymie - du grec tropos "manière, mode" - lie un lexème verbal superordonné (ex : entrer) à un lexème sémantiquement apparenté quoique plus spécifique (ex : s'introduire). Elle se substitue, dans le domaine des verbes, à la relation d'hypéronymie.

3. Dans de nombreux cas, la classification des verbes des listes A et B a été précédée d'une activité individuelle de production de phrases comprenant ces lexèmes (cf. section 4). Par ailleurs, diverses séances de travail par groupes et de mise en commun des classifications ont été filmées (Gangneux \& Garcia-Debanc, 2013).

4. Pour compléter cette brève analyse des prédicats de déplacement strict et des interactions entre changements de relation et changements d'emplacement, précisons qu'un certain nombre de verbes de simple changement d'emplacement (voir tableau 1) peuvent, en présence d'un groupe prépositionnel (GP) spatial adéquat, donner lieu à une lecture dans laquelle un changement de relation (final) s'ajoute au changement d'emplacement initialement dénoté par le verbe (ex: Max a couru/rampé/reculé/glissé dans le jardin). Les prédicats de changement d'emplacement concernés présentent des traits sémantiques bien spécifiques - vitesse, opposition à une force, direction/déplacement linéaire orienté, entrainement par une force - qui ont été regroupés au sein de la notion de «tendancialité » (Aurnague, 2008, 2011). 
5. CE2A : 3 élèves ont produit entre 0 et 5 verbes de déplacement et 5 autres 6 à 10 verbes. CM1$\mathrm{CM} 2$ : les 22 élèves ont produit entre 1 et 5 verbes de déplacement.

6. Voici, pour chacune des classes, les verbes de déplacement autonome relevés dans les productions libres, une fois ordonnés par nombre d'occurrences (entre parenthèses). CE2A : accélérer, arriver, escalader, galoper, glisser, nager, naviguer, monter, partir, planer, se promener, survoler (1) < entrer/rentrer, se frayer un passage, passer + Prép. (2) < aller + Prép. (4) < se rapprocher, voler (5) < courir, marcher (7) [+ faire de la luge/de l'équitation/des pas chassés/du sport/du vélo (1)]. CM1-CM2 : se déplacer, descendre, s'enfuir, nager, plonger, rentrer, retourner, venir, voler $(1)<$ marcher, monter $(2)<$ courir, s'échapper (3) < partir (4) < aller + Prép. (14) [+ sauter, prendre le car (1)]. Un très petit nombre de verbes de déplacement causé (voir section 3 ) a été recensé et il a, pour cette raison, été laissé à l'écart du comptage : déplacer (CE2A), amener/emmener (CM1-CM2).

7. Il est possible que certaines "erreurs" soient imputables à la combinaison des deux contraintes mobilisées par cette activité/tâche, c'est-à-dire à la nécessité de "contrôler » simultanément le concept de déplacement strict et les divers emplois des verbes. La charge cognitive serait, d'une certaine manière, plus lourde ici que dans la production libre de phrases.

8. Une seule différence générale a été notée dans cette activité (production de phrases dirigée) entre les élèves de CE2A et ceux de CM1-CM2. Elle concerne la variété des temps verbaux qui est plus grande dans le second cas puisque, outre le présent, sont également utilisés le passé composé, l'imparfait et le futur. Cette différence était déjà perceptible dans la tâche de production libre (malgré le nombre inégal de données recueillies pour les deux classes). Il en est de même dans les textes narratifs.

9. L'orthographe des exemples a généralement été rétablie.

10. Alors que les divers écarts syntaxiques mentionnés se retrouvent dans les deux classes, les constructions transitives recensées en CM1-CM2 ne portent que sur aboutir et accéder (pas sur parvenir). Au sujet d'aboutir, notons qu'en dépit des apparences, ce verbe présente, dès le XIV ${ }^{\mathrm{e}}$ siècle et jusqu'à nos jours, un emploi spatial majeur indiquant qu'une cible (terre, fossé, bâtiment et, plus tard, chemin) confine à ou touche un site (ce sens a été longtemps partagé avec le verbe abouter, de même étymon; cf. TLF) : Le chemin aboutit à l'école (CE2A). Cet usage du verbe est sans doute à l'origine des emplois, souvent minimisés ou passés sous silence, dans lesquels une cible autonome (typiquement animée) parcourt une entité matérielle "étendue » (habituellement de type « chemin ») pour terminer son déplacement dans un site : Nous allons aboutir dans un cul-desac (CM1-CM2). À partir du XIX ${ }^{\mathrm{e}}$ siècle, ces deux emplois spatiaux sont parmi les plus courant du verbe, avec une troisième acception exprimant le fait d'« arriver à un résultat/terme, achever, réussir ", en construction absolue notamment : Marsy, moyennant le million, s'engage à m'appuyer et à faire aboutir ma demande dans le délai d'un mois (Zola, Son Excellence Eugène Rougon, 1876 ; cité par le TLF). Le TLF qualifie de "figurée » cette dernière acception et relie son émergence à l'idée de route et de cheminement.

11. Arriver n'inclut aucune de ces contraintes sémantiques supplémentaires et conserve donc une forme de « neutralité » par rapport à elles.

12. Dans le champ du déplacement autonome, aboutir dénote le guidage de la cible par un site « intermédiaire» (menant au site «final») et, indirectement (i.e. d'un point de vue pragmatique), la non-intentionnalité du déplacement. L'affectation du site intervient dans ce type de procès à travers la notion de contact/«transmission d'énergie minimale » (voir abouter, toucher; Vandeloise, 2001 : 270-302) et celle de la cible est la conséquence du guidage/contrôle. Accéder et parvenir (comme atteindre) sont, quant à eux, sous-tendus par le concept d'obstacle/ difficulté « externe » auquel s'oppose l'intention de la cible d'atteindre le site final ou d'avancer (au maximum) dans une direction donnée. L'opposition possiblement exercée par la cible vis-àvis d'un éventuel obstacle et l'atteinte du site font que ce dernier se trouve affecté par la réalisation du procès (l'affectation simultanée de la cible n'est pas exclue mais elle paraît souvent reléguée à un second plan). 
13. Dans eManulex (Ortéga \& Lété, 2010), la fréquence estimée d'Usage par million de mots (U), appliquée au lexique des lemmes extraits des manuels de CE2-CM2, montre qu'arriver est nettement plus utilisé qu'atteindre - parvenir et, surtout, aboutir et accéder présentant des scores encore plus faibles. Sur cette base, on devrait s'attendre à ce que les élèves ayant des difficultés à manier aboutir, accéder et parvenir calquent presque exclusivement leur usage sur celui d'arriver (d'autant plus que ce prédicat apparait dans la production de plus de $80 \%$ des enfants avec une réussite supérieure à $90 \%$, à la différence d'atteindre). Or ce n'est pas ce qui se produit. Tous ces verbes relevant de la même catégorie en termes de schéma spatiotemporel sous-jacent (voir plus haut), c'est donc vers leurs propriétés sémantiques additionnelles qu'il faut se tourner au moment de rendre compte de ce comportement. Alors qu'arriver est indifférent à ces propriétés (il ne les inclut pas dans son sémantisme ; cf. note 11), les autres verbes - et, tout spécifiquement, accéder, parvenir et atteindre - les partagent largement.

14. Lorsque le seuil est abaissé à $70 \%$ - plus de $70 \%$ d'élèves produisent plus de $70 \%$ d'emplois corrects -, 6 verbes supplémentaires viennent s'ajouter à ceux déjà listés pour le CE2A : accéder, s'enfuir, quitter, se rendre, sortir, venir. Un seul nouvel item s'agrège à la liste en CM1-CM2 : atteindre

\section{RÉSUMÉS}

Ce travail interroge les articulations entre modélisations linguistiques en sémantique et données recueillies auprès de classes de cycle 3 d'école primaire française (élèves de 9-12 ans), dans le cadre d'un projet de recherche collaborative. Le protocole se propose d'évaluer les effets d'activités de classification de verbes en étude de la langue sur la diversité et la pertinence de leur emploi, dans la rédaction de récits impliquant de la localisation «dynamique ». Les verbes visés sont des verbes intransitifs ou transitifs «indirects» du français se référant à des déplacements autonomes (i.e. non causés ; ex : aller + Prép., arriver, entrer, partir, se rendre, sortir), pour lesquels une nouvelle caractérisation sémantique a été mise au point. Les procès dénotés par ces verbes ont une structure spatiotemporelle qui associe deux concepts - changement d'emplacement et changement de relation locative élémentaire - et se singularisent notamment par la « polarité » initiale ou finale du changement de relation (locative élémentaire) sous-jacent. Les verbes de déplacement strict ainsi isolés se distinguent des verbes de déplacement au sens faible étudiés par ailleurs, dont le sémantisme ne comprend qu'un simple changement d'emplacement (ex : foncer, grimper, marcher, reculer).

Après la description du protocole de recueil de données et la présentation de la modélisation linguistique, l'article examine les résultats obtenus, qu'ils concernent la production de phrases ou la classification des verbes de déplacement strict. L'analyse permet de mettre en évidence les difficultés rencontrées par les élèves dans la construction syntaxique de certains verbes ainsi que l'accessibilité à des concepts réputés abstraits comme celui de polarité, intuitivement retenu par les élèves dans leurs classifications.

This work deals with the articulation between linguistic modelling in semantics and data collected in classrooms of French primary school (9 to 12 year-old pupils), within the framework of a collaborative project. The protocol aims at assessing the effects of activities of verbs' classification implemented as language training, on the variety and relevance of verbs' uses when writing a story that involves "dynamic" location. The verbs under examination are 
intransitive or "indirect" transitive verbs of French referring to autonomous motions (i.e., noncaused motion; e.g., aller + Prep. 'to go + Prep.', arriver 'to arrive', entrer 'to go in, to enter', partir 'to go (away), to leave', se rendre 'to go to', sortir 'to go out'), for which a new semantic characterization has been proposed. The processes denoted by these verbs have a spatiotemporal structure that combines two concepts -change of placement and change of basic locative relation- and are distinctive for the initial or final "polarity" of the underlying change of (basic locative) relation, among other things. The verbs of strict motion thus defined differ from verbs of motion in the weak sense studied elsewhere, as their semantic content only includes a change of placement (e.g., foncer 'to tear along', grimper 'to climb', marcher 'to walk', reculer 'to (move) back').

After having described the data collection protocol and set out the linguistic modelling, the paper examines the results obtained for both sentence production and classification of strict motion verbs. The analysis allows us to highlight the difficulties related to the syntactic construction of specific verbs as well as the access to supposedly abstract concepts like polarity that are intuitively selected by students in their classifications.

\section{INDEX}

Keywords : teaching of French as a first language, vocabulary teaching, verbs of strict motion, sentence production, classification of verbs

Mots-clés : didactique du français langue première, enseignement du lexique, verbes de déplacement strict, production de phrases, classification de verbes

\section{AUTEURS}

\section{MICHEL AURNAGUE}

Université Toulouse - Jean Jaurès, Centre national de la recherche scientifique, CLLE-ERSS, UMR 5263, F-31058, France

\section{CLAUDINE GARCIA-DEBANC}

Université Toulouse - Jean Jaurès, Centre national de la recherche scientifique, CLLE-ERSS, UMR 5263, F-31058, France

Espé Toulouse Midi-Pyrénées 